\title{
An Application of Artificial Bee Colony Algorithm for Reservoir Optimization: A Case Study of Chenderoh Dam, Malaysia
}

\author{
Shi-Mei Choong ${ }^{1}$, Prof. A. El-Shafie ${ }^{2}$ and Dr. Wan Mohtar W.H.M. ${ }^{1}$
}

\begin{abstract}
Reservoir operators commonly manage and regulate the downstream water flow and the demands over time and space. It becomes a challenging task for the decision maker to evaluate the trade-off during high inflow and low inflow season especially for multipurpose hydropower system. The main goal of the present study is to develop a set of release policy under three different inflow scenarios (high, medium and low) for the Chenderoh hydropower reservoir using the Artificial Bee Colony algorithm. Due to its capability to identify different possible events happened in the reservoir, the proposed algorithm provided promising solutions which minimize the water deficit in the operating system. Based on the thought of refined performance by ABC model, three indices criteria (reliability, resilience and vulnerability) are used to evaluate its effectiveness. ABC model has provided good results in terms of reliability (63.9\% meeting the demand, $11.8 \%$ more than the demand and $24.3 \%$ less than the demand) and small vulnerability (1.31) leading to develop an outstanding release policy for optimal operation.
\end{abstract}

Keywords-Reservoir Optimization, Artificial Bee Colony, Simulation, Release Policy.

\section{INTRODUCTION}

Water management for a hydropower system is nonlinear with the respect to their dynamics, operation constraints and objectives. These inherent variables are leading to form numeric optimization methods from linear programming to dynamic programming in solving the reservoir operation problem. Nevertheless, these renders classical methods cannot deal with the uncertainty of future conditions such as inflows which make the results irrelevant to the reality (Choong \& ElShafie, 2014). Therefore, only probabilistic approaches offer a scientifically rigorous method to cope with the future uncertainty.

Artificial Bee Colony (ABC) algorithm is among the most popular evolution algorithm to solve the multi-objective problems. It mimics the honey bee swarm intelligent behaviour in solving numerical functional optimization. The algorithm simulates the foraging behaviour of the honey bee in identifying and exploitation the infeasible region of the search area. ABC algorithm was developed by Karaboga (2005) which inspired many researchers to do further improved and

\footnotetext{
${ }^{1}$ Civil and Structural Engineering Department, National University of Malaysia, Malaysia

${ }^{2}$ Civil Engineering Department, University of Malaya, Malaysia
}

applied it to various problems. Karaboga and Bastuk (2007a, 2007b) allocated ABC algorithm on multidimensional task and compared its performance with the performances of Particle Swarm Optimization (PSO), Particle Swarm Inspired Evolutionary Algorithm (PS-EA) and Genetic Algorithm (GA). The authors satisfied with its ability to get out of local minimum and its effective application on multivariable function optimization. Hossain et al. (2013) proposed ABC algorithm to derive operating policies for a multi-purpose reservoir system and demonstrated its applicability and low computation complexity through a case study of Aswan High Dam, Egypt. On the other hand, Zhou et al. (2014) employed the new multi-objective ABC algorithm and applied for shortterm scheduling of hydrothermal system. According to the results, the proposed algorithm has a better convergence ability which is feasible as a powerful tool in the field of reservoir optimization.

Notably, the tasks are getting more challenging when in the water resource management; decisions are made with the reference to the future and most probably will always be unknown. To overcome the shortcomings for the decision maker in regulating the downstream water flow and demands over time and space, this paper presents an application of $\mathrm{ABC}$ algorithm in search of optimal release for Chenderoh Reservoir. The existing nonlinear swarm optimization algorithm, $A B C$ is ready to adopt and incorporate with the known (historical reservoir inflow) input for the model to provide possible solutions (release policy). An optimal water distribution relation needs to be maintained between the water release and reservoir storage in every operation time period (Hossain \& El-shafie, 2013). Therefore, the main objective of this paper is to minimize the water deficit in the operating system.

\section{CASE STUDY: CHENDEROH DAM}

Chenderoh Reservoir is located in the state of Perak of Peninsular Malaysia ( $4^{\circ} 57^{\prime} 42^{\prime \prime}$ North and $100^{\circ} 58^{\prime} 38^{\prime \prime}$ East). It is the oldest dam in Malaysia with Hollow Buttress type founded on granite and being commissioned for hydropower generation, domestic water supply and irrigation. There are four cascading reservoirs that found along the Perak River Temenggor, Bersia, Kenering and Chenderoh Reservoir that found from upstream to downstream respectively as shown in Figure 1 (Bu \& Seng, 1997). Due to the relative position of the reservoirs in the cascading system, the inflow rate of the two 
newer reservoirs that located at the most upstream (Temenggor and Bersia) relies on the catchment characteristic condition. While the major inflow rate of the downstream reservoirs (Kenering and Chenderoh) depends on the release of the preceding reservoirs (Asfaw et al. 2013). Hence, a total thirteen years (2000-2012) of consecutive historical inflow data (preceding reservoirs' output) has been used as the input for the ABC optimization model. In order to provide the optimal release under different events of the actual inflow, these inflow data are categorized into three discrete regions: high inflow, medium inflow and low inflow as given in Figure 2. The dam is $20 \mathrm{~m}$ height with a total capacity of $94.50 \mathrm{Mm}^{3}$ supply a huge quantity of water division for maximum power generation about $38 \mathrm{MW}$. Therefore, the Chenderoh reservoir operation rules should follow its storage range and release bound. The storage range for the operating policy is taken from $45 \mathrm{Mm}^{3}$ to $94.50 \mathrm{Mm}^{3}$ while the weekly release bound is between $5 \mathrm{Mm}^{3}$ and $25 \mathrm{Mm}^{3}$.

\section{ARtificial BeE Colony Algorithm (ABC)}

The ABC algorithm can be described in four phases which including the initialization, employed bee phase, onlooker bee phase and scout bee phase. Formally, each population of a solution is represented by the potential food source while the solution quality corresponds to the food source quality. It is assumed that the number of the food source around the hive, the number of employed bee and the number of the onlooker bee are equal and there is only one scout bee in the $A B C$ algorithm. The employed bee is the bee that exploits the food source (possible solution) from the specified region of the search area. When the employed bee arrives to the hive, it might have three options to choose, either it goes back to the source and keep continuing to collect, go to the dance floor and share the information about the food source by waggle dance or becomes a scout bee when the source is depleted. The onlooker bee is the bee that stays in the hive while waiting for the information brings by the employed bee. Good quality of food source is preferred instead of the bad one and memorized by the onlooker bee to exploit. The scout bee is the bee that employed to search for a new food source randomly once their food source has been fully exploited and abandoned. The foraging route as illustrated in Figure 3 is repeated until the termination conditions are fulfilled which is the population size (colony size) and the maximum generation number (cycle number. The algorithm underlying a standard ABC is outlined in Figure 4 (Choong \& El-Shafie, 2015).

The setup of food source in the ABC algorithm for the bee as the searching space for optimum releases is mathematically expressed as Equation (1). A set of release options which consisted of 52 values for each month was taken as a food source. That means a single source contained 12 consecutive monthly release options.

$$
y_{i}(i=1,2, \ldots, S)
$$

Where, $y_{i}$ is the $i^{\text {th }}$ member of a single food source which denotes the population size of $S$. The fitness values are then computed and the best food source among the population is recorded. Employed bee tries to improve its location by using local search step in every iteration (Diwold et al. 2011) as given in Equation (2). The amount of the new source is equal to half of the total food source.

$$
x_{i j}=y_{i j}+\theta_{i j}\left(y_{i j}-y_{k j}\right)
$$

Here, $x_{i j}$ represents the new updated value of the release for $i^{\text {th }}$ location of $j^{\text {th }}$ source and $j$ is the dimensional vector, yij corresponds to the current single release of $j^{\text {th }}$ source. $\theta_{i j}$ is taken as a random number between -1 and 1 while $y_{k j}$ denotes other randomly chosen release and must take from the different neighbour source. The dimensions to be altered are randomly chosen. The fitness value of the new release is checked again with the optimum solution and the decision for the survival of a particular source depends on the fitness of the solutions.

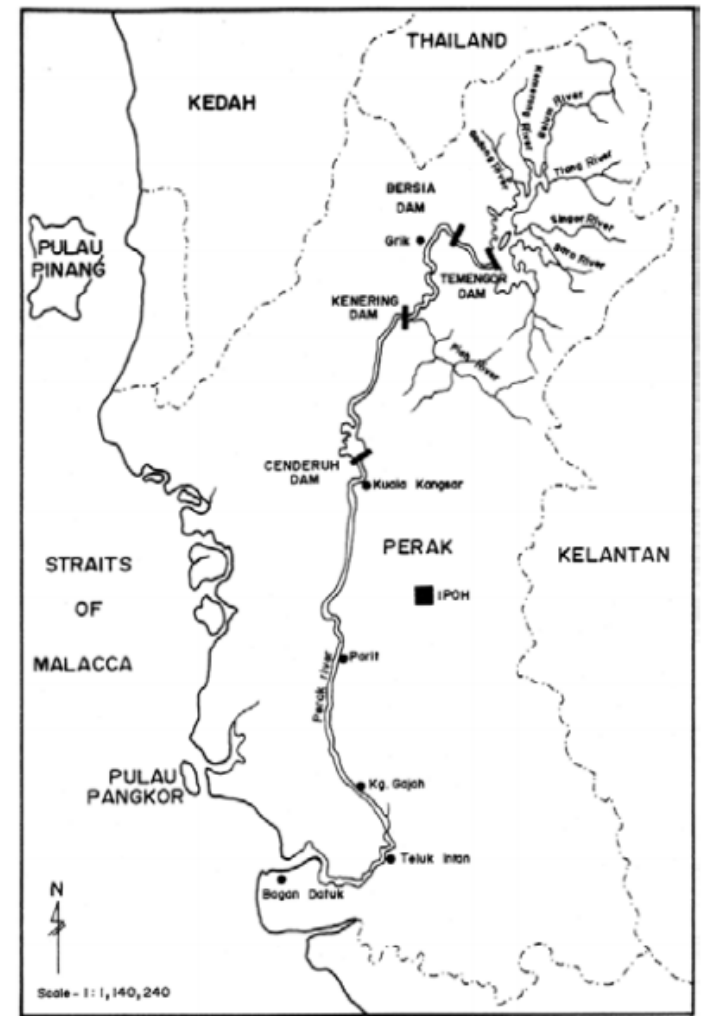

Fig. 1: Location Map and Layout of the Reservoirs along Perak River.

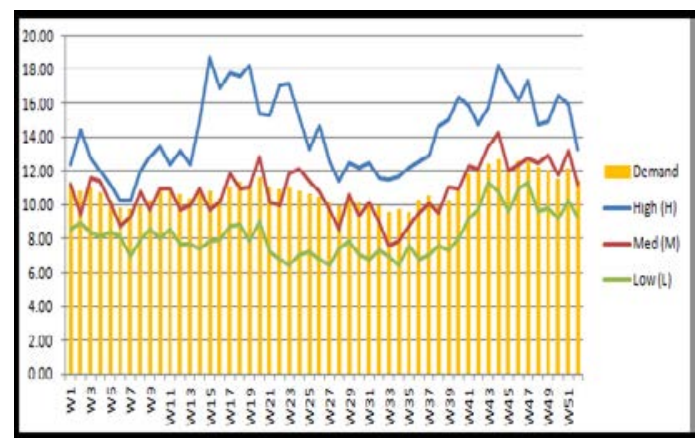

Fig. 2: Three Discrete Inflow States with Monthly Demand (in $\mathrm{Mm}^{3}$ ). 


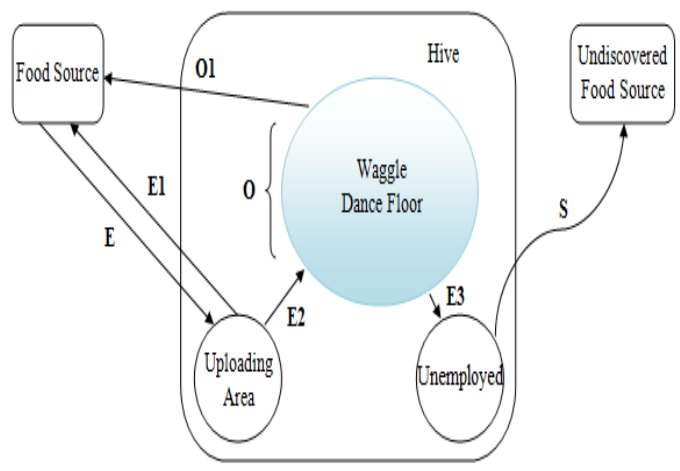

Fig. 3: Honey Bee Foraging Process.

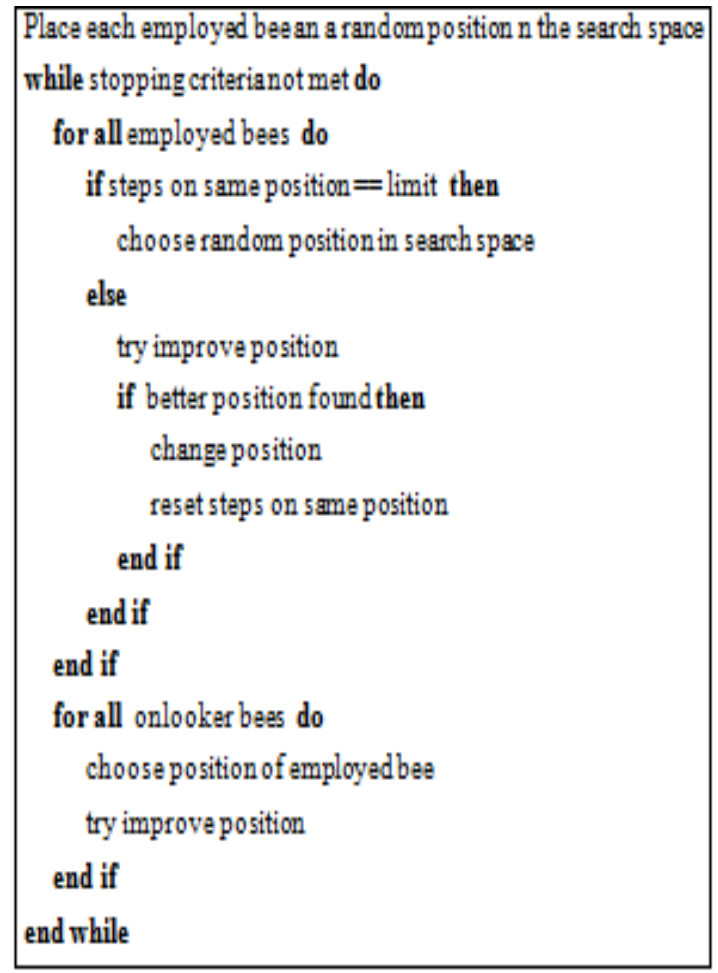

Fig. 4: The ABC Algorithm.

After employed bee has updated its position, onlooker bee chooses the food source according to their probability of being selected. A standard roulette wheel selection (Eiden \& James, 2003) as expressed in Equation (3) is used and better solutions attract more onlooker bees.

$$
P_{i}=\frac{f\left(y_{i}\right)}{\sum_{j=1}^{N} f\left(y_{j}\right)}
$$

Now, the probability $\left(P_{i}\right)$ of any $i^{\text {th }}$ source is the ratio of its individual fitness to total fitness of all the other sources, where $\mathrm{N}$ is the total number of the food source. Then, the onlooker improved the solution using the same mechanism again as outlined in the Equation (2) if a better solution is found. Soon and later, the scout bee needs to find the new food source randomly to replace the abandoned source which is the source that poses weak fitness value.

\section{MODEL IMPLEMENTATION AND VALIDATION}

The objective function is given by Equation (4). During the course of evolving the optimal solution for the reservoir, ABC algorithm is projected to explicit the constraints of the storage and release volumes. The proposed algorithm is then applied to the consecutive weekly water balance relation as given in Equation (5) in order to define a new set of possible release policy which satisfies the solution constraints.

$$
\begin{aligned}
\min f(x) & =\sum_{t=1}^{52}\left(R_{t}-D_{t}\right)^{2} \quad t=1,2, \ldots, 52 \\
S_{(t+1)} & =S_{t}+I_{t}-R_{t}-O_{t}-L_{t}
\end{aligned}
$$

In addition, the reservoir maintenance constraints in this study are expressed in the flowing section.

- Reservoir water storage constraints: $45 \leq S_{t} \leq 94.50$

- Reservoir overflows constraints: $O_{t}=S_{t+1}-94.50$

- Reservoir release constraints: $5 \leq R_{t} \leq 25$

Where, $R_{t}$ denotes the release from the reservoir; $D_{t}$ represents the demand for a time period $t ; S_{(t+1)}$ is the final reservoir storage during the time period of $t ; S_{t}$ is the initial reservoir storage in period $t ; I_{t}$ denotes the total inflow from the preceding reservoirs and the direct inflow into the reservoir in period $t ; O_{t}$ represents the overflow from the reservoir in period $t$ and $L_{t}$ denotes the loss from the reservoir which comprises the evaporation, seepage water losers and so on in period $t$, whose unit is Mm3.

The penalty functions expressed in Equation (9) and (10) are very important in the application of $\mathrm{ABC}$ optimization model. It is used to manage the reservoir bounds and the listed constraints.

$$
\begin{aligned}
& \text { Penalty_1 }=\left\{\begin{array}{r}
0, \text { if } S_{t}>S_{\min } \\
C_{1}\left(S_{\min }-S_{t}\right)^{2}, \text { if } S_{t}<S_{\min }
\end{array}\right. \\
& \text { Penalty_2 }=\left\{\begin{array}{r}
0, \text { if } S_{t}<S_{\max } \\
C_{2}\left(S_{t}-S_{\max }\right)^{2}, \text { if } S_{t}>S_{\max }
\end{array}\right.
\end{aligned}
$$

Where, $C_{1}$ and $C_{2}$ are the penalty coefficients for the reservoir. The reservoir water storage in any time of period $t$ should not exceed the maximum storage $\left(S_{\max }\right)$ and should not be less than the minimum storage $\left(S_{\min }\right)$.

In calibrating the release policy, simulation has been done to perform the risk analysis for the model. Three different indices criteria (reliability, resilience and vulnerability) are used in order to evaluate the effectiveness of the optimization model. The definition of these three indices in measuring the level of performances are conducted by Moy et al. (1986). The performance indices are mathematically expressed as follow:

- Volumetric Reliability: $R_{v}=\left(\frac{v}{v}\right) \times 100 \%$ 
- Periodic Reliability: $R_{\mathrm{p}}=\left(\frac{n}{N}\right) \times 100 \%$

- Resilience: $R_{\mathrm{s}}=N S / N T$

- Vulnerability: $V=\frac{\sum_{t=1}^{i v}\left[\max \left(D_{t}-R_{t}\right)\right]}{m} \quad t=1,2, \ldots N$

Where, $v$ is the volume of water deficit; $V$ represents the volume of targeted demand; $n$ denotes the total number of time periods meeting the demand; $N$ is the total number of considered time period; NS denotes the number of satisfied time period followed by a failure; $N T$ represents the number of total failure period and $\mathrm{m}$ indicates the number of model failure period in the simulation.

\section{RESULTS AND DISCUSSION}

For an optimal algorithm, its convergence curve is very vital because it symbolized the stability and capability for such system to pursue the optimal state through the iteration. Figure 5 shows the convergence curves for the objective function values that achieved through 1000 iteration for the three different inflow state at minimum initial storage of $45 \mathrm{Mm}^{3}$. It should pose the minimum fitness value of any iteration. At this juncture, $A B C$ optimization models manage to reach the minimum fitness within 30 iterations in achieving the optimal release for the reservoir following the optimal strings.

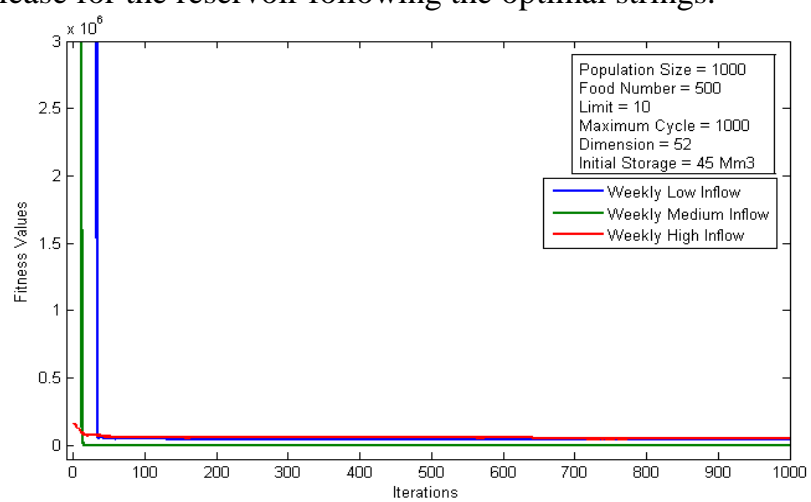

Fig. 5: Fitness Values of all Iterations.

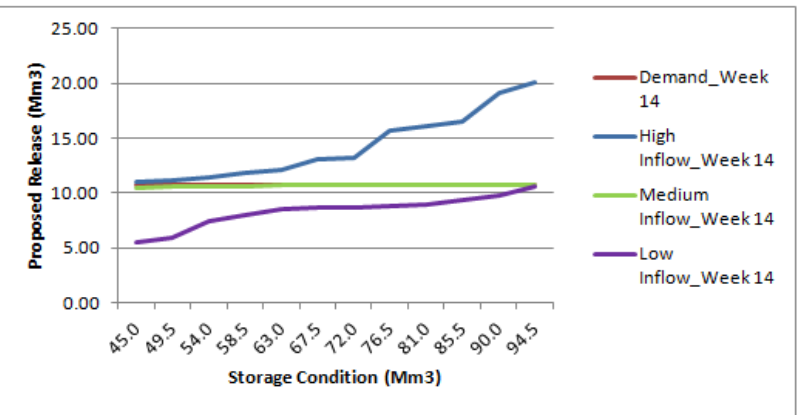

Fig. 6: Release Curve for the Week 14

A total 156 of weekly release curve have been developed under three different inflow scenarios. The variant of the reservoir release for different policies were extracted as illustrated in Figure 6 for the Week 14. Due to the fact that the reservoir level is usually above the upper limit during high inflow period and the water supplied to the hydropower generation is always maximized, water released from the reservoir is always more than the demand. On the other hand, almost all of the releases can meet the demand during medium inflow period even in low storage capacity. However, water released from the reservoir is hardly meeting the exact demand during critical low inflow period. From a cursory analysis of the release curves, the water shortage is very apparent during low storage capacity period. This recognized the relation between low inflow and the corresponding water release of the reservoir is one of the most important issues which hydrologists and engineers have been busy with. With the proposed release policy in such a way, the decision maker may have a clear understanding in deciding on the release amount to minimize the water deficit under different scenario.

As mentioned earlier, a simulation that associated with the reservoir operation is based on the water balance continuity equation as presented in Equation (5). The whole simulation process started with a predefined initial storage volume and its historical inflow amount for a considered data series. The targeted demand is used to access the optimal results. Optimal release is then obtained and the final storage became the initial storage for the next consecutive week correspondingly. The process continued until the end of the considered historical data series. Figure 7 shows the simulated releases with the demand for weekly purpose. The results from the simulation have further been applied for performances indices to validate its effectiveness which presents in Table 1.

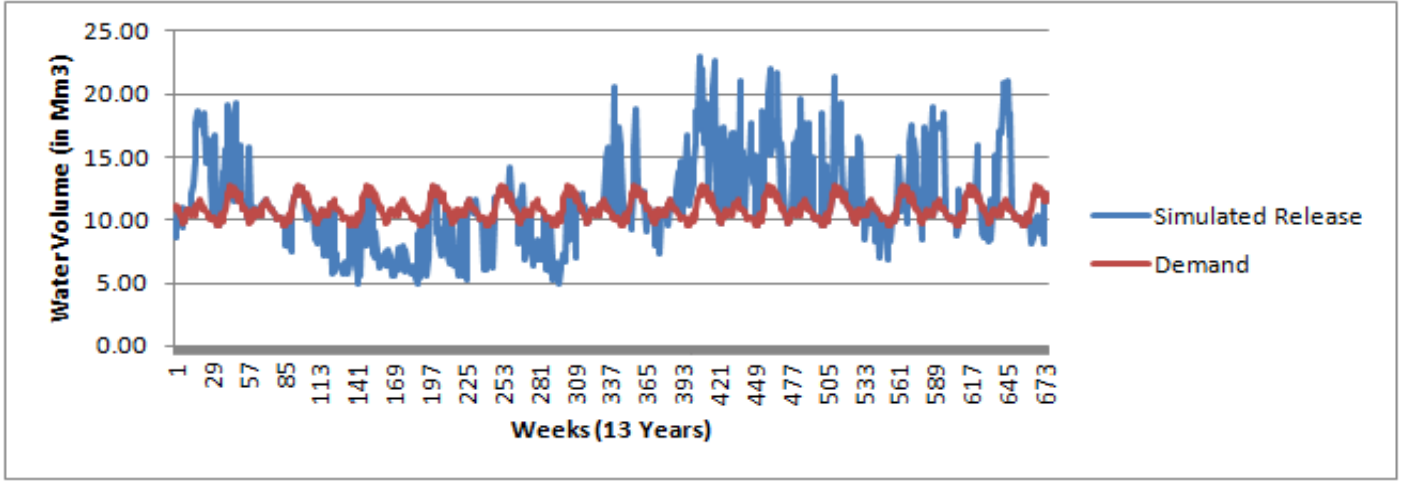

Fig. 7: Simulated Release with Targeted Demand 
TABLE 1: MODEL PERFORMANCE INDICES

\begin{tabular}{llll}
\hline \hline & & ABC Optimization Model (Total 676 release) \\
\hline Reliability & Periodic & More than demand & $11.8 \%$ (80 times) \\
& & Meet than demand & $63.9 \%$ (432 times) \\
& Less than demand & $24.3 \%$ (164 times) \\
Resilience & Volumetric & & $95.7 \%$ \\
& Condition & 0.18 \\
& Max. no. of & 44 \\
& consecutive & \\
Vulnerability & shortage & \\
\hline \hline
\end{tabular}

The validation results of the model give $95.7 \%$ of agreement for the optimization performance. That is to say that, the optimum release developed by ABC model under different dependence inflow scenarios will be very helpful in assessing the reservoir operation. Results suggested that ABC model provided a better quality of reliability (63.9\% meeting the demand, $11.8 \%$ more than the demand and $24.3 \%$ less than the demand) with very good vulnerability (1.31). Certainly, this summarized that the proposed ABC optimization model competent to used as a feasible tool in the reservoir operation management.

\section{CONCLUSION}

Seeking an optimal operation strategy for the reservoir is very important for generating the hydropower and making the full utilization of water resource. A reliable release policy under different inflow scenarios was developed for the reservoir operation by adopted the proposed ABC algorithm. ABC model deduced with the reservoir constraints to produce satisfactory simulation results. The result was verified based on reliability, resilience and vulnerability indices. The overall outcome proved that the proposed algorithm is very supportive in enhancing the water management for the reservoir optimization operation which incorporates by the decision maker. In addition, ABC model is a user-friendly method which has low complexity during computation and well established. Therefore, it is having a great potential as a powerful tool in solving the reservoir optimization problem.

\section{ACKNOWLEDGMENT}

The authors would like to express sincere appreciation to the Generation Department of Tenaga Nasional Berhad Malaysia (TNB) for proving the relevant data and their kind cooperation in this study.

\section{REFERENCES}

[1] Asfaw, T. D., Yusof, K. W., \& Hashim, A. M. (2013). Competenceoriented decision model for optimizing the operation of a cascading hydroelectric power reservoir. [Article]. Research Journal of Applied Sciences, Engineering and Technology, 6(10), 1895-1901.

[2] Choong, S. M., \& El-Shafie, A. (2014). State-of-the-Art for Modelling Reservoir Inflows and Management Optimization. [Review]. Water Resources Management, 29(4), 1267-1282. http://dx.doi.org/10.1007/s11269-014-0872-z

[3] Choong, S. M., \& El-Shafie, A. (2015). Artificial Bee Colony (ABC) Approach for Reservoir Operation. International Journal of Advances in Mechanical and Civil Engineering, 2(1), 5-9.
[4] Diwold, K., Beekman, M., \& Middendorf, M. (2011). Honeybee Optimisation - An Overview and a New Bee Inspired Optimisation Scheme. In B. K. Panigrahi, Y. Shi \& M.-H. Lim (Eds.), Handbook of Swarm Intelligence: Concepts, Principles and Applications (pp. 295327). Berlin, Heidelberg: Springer Berlin Heidelberg. http://dx.doi.org/10.1007/978-3-642-17390-5_13

[5] Eiben A.E. , J. E. S. (2003). Introduction to Evolutionary Computing, Springer-Verlag Berlin Heidelberg

[6] Hla Bu, S. S., \& Seng, L. T. (1997). Fish parasite communities in tropical reservoirs along the Perak River, Malaysia. [journal article]. Hydrobiologia, 356(1), 175-181. http://dx.doi.org/10.1023/A:1003158700538

[7] Hossain, M. S., \& El-shafie, A. (2013). Performance analysis of artificial bee colony (ABC) algorithm in optimizing release policy of Aswan High Dam. Neural Computing and Applications, 1-8.

[8] Karaboga, D. (2005). An idea beased on honey bee swarm for numerical optimization: Erciyes University, Engineering Faculty, Computer Engineering department.

[9] Karaboga, D., \& Basturk, B. (2007a). Artificial Bee Colony (ABC) Optimization Algorithm for Solving Constrained Optimization Problems. In P. Melin, O. Castillo, L. Aguilar, J. Kacprzyk \& W. Pedrycz (Eds.), Foundations of Fuzzy Logic and Soft Computing (Vol. 4529, pp. 789-798): Springer Berlin Heidelberg. http://dx.doi.org/10.1007/978-3-540-72950-1_77

[10] Karaboga, D., \& Basturk, B. (2007b). A powerful and efficient algorithm for numerical function optimization: artificial bee colony (ABC) algorithm. Journal of Global Optimization, 39(3), 459-471. http://dx.doi.org/10.1007/s10898-007-9149-x

[11] Moy, W. S., Cohon, J. L., \& ReVelle, C. S. (1986). A Programming Model for Analysis of the Reliability, Resilience, and Vulnerability of a Water Supply Reservoir. [Article]. Water Resources Research, 22(4), 489-498. http://dx.doi.org/10.1029/WR022i004p00489

[12] Zhou, J., Liao, X., Ouyang, S., Zhang, R., \& Zhang, Y. (2014). Multiobjective artificial bee colony algorithm for short-term scheduling of hydrothermal system. International Journal of Electrical Power \& Energy Systems, 55, 542-553. http://dx.doi.org/10.1016/j.ijepes.2013.10.013 